\title{
Impactos das mudanças climáticas na demanda de irrigação da bananeira na Bacia do Jaguaribe
}

\author{
Rubens S. G ondim ${ }^{1}$, Marco A. H. de Castro ${ }^{2}$, \\ Adunias dos S. Teixeira ${ }^{2} \&$ Sílvio R. de M. Evangelista ${ }^{3}$
}

\begin{abstract}
RESUMO
As mudanças climáticas têm potencial de alterar os processos do ciclo hidrológico, tais como precipitação, que afeta o escoamento superficial, temperatura e umidade relativa do ar devido à sua estreita relação com evaporação e vazão em corpos hídricos e evapotranspiração das plantas. 0 objetivo do presente trabal ho foi analisar os impactos das mudanças climáticas na necessidade hídrica da cultura da bananeira, considerando-se cenários de mudanças climáticas em escala local, em nível de bacia hidrográfica (rio Jaguaribe, no trecho compreendido entre as barragens do Castanhão e de Itaiçaba). U m conjunto composto de uma baseline (climatologia de base) do modelo de 1961-1990 e de projeções climáticas, foi processado. $\mathrm{O} s$ dados foram então extraídos considerando-se as coordenadas geográficas da região em estudo, com resolução de $0,44 \times 0,44^{\circ}$, a fim de abranger toda a área. Estimou-se a evapotranspiração de referência (ETO) através de dados da temperatura média mensal e se projetou uma elevação na necessidade hídrica bruta média anual para 2040 com relação às condições iniciais, de $1.989 \mathrm{~mm}$ para $2.536 \mathrm{~mm}$ e $2.491 \mathrm{~mm}(27,50$ e $25,24 \%)$ para os cenários A2 e B2, elaborados pelo Painel Intergovernamental de Mudanças Climáticas, respectivamente.
\end{abstract}

Palavras-chave: evapotranspiração, irrigação, SIG

\section{Impact of climate change on irrigation requirement of banana in Jaguaribe river Basin, Ceará, Brazil}

ABSTRACT

Climate change has a potential to impact hydrologic cycle processes, such as rainfall, which affect runoff, temperature and air humidity that have relationship to evaporation over water bodies and plant evapotranspiration. The purpose of this study was to assess impacts of climate change on irrigation water demand of banana, at the river basin level (Jaguaribe river,

Embrapa Agroindústria Tropical. Rua Dra. Sara Mesquita 2270. CEP 60511-110, Fortaleza, CE. Fone: (85) 3391-7214. E-mail: rubens@cnpat.embrapa.br

2 DEHA/U FC. Av.M ister Hull s/n. Bloco 713 e DEN A/U FC. Bloco 804. CEP 60455-760, Fortaleza, CE. Fone: (85) 3366-9492. E-mail: marco@ufc.br. Fone: (85) 3366-5966. E-mail: adunias@ufc.br

${ }^{3}$ Embrapa Informática Agropecuária. Av. André Tosello 209-Barão Geraldo. C.P. 6041, CEP 13083-886, Campinas, SP. Fone: (19) 3789-5740. E-mail: silvio@cnptia.embrapa.br 


\section{INTRODUÇÃO}

Hegerl et al. (2007) chamam a atenção não apenas para o entendimento de mudanças climáticas que resultam de forçantes externas antropogênicas e se diferenciam das naturais, mas também para o fato de poderem ser distinguidas das mudanças e variabilidades que resultam de processos internos do sistema climático.

Em geral, na sua maioria, os Modelos de Circulação Global simulam processos na escala global ou continental em detalhe e provêm uma representação de precisão razoável da média climática planetária (Dibike \& Coulibaly, 2005), embora incapazes de representar características locais.

Redução de escala dinâmica envolve o aninhamento de um Modelo de Circulação Regional (RCM) a uma resolução mais grosseira de um Modelo de Circulação Global (GCM). Um RCM utiliza o GCM para definir as condições de contorno atmosféricas variáveis com o tempo, em torno de um domínio finito no qual a dinâmica física da atmosfera é modelada, utilizando-se uma malha horizontal espaçada $20-50 \mathrm{~km}$. A principal vantagem de RCMs é sua capacidade de solução de características atmosféricas de escala mais detalhada (Wilby et al., 2002).

Binder (2006), recomendou detalhar mais os cenários disponíveis de mudanças climáticas para a escala de bacia hidrográfica utilizando-se ainda modelos hidrológicos, a fim de se estudar impactos das mudanças climáticas nos recursos hídricos.

Schlenker et al. (2007), analisando o impacto da mudança climática na agricultura irrigada na Califórnia concluíram que, para climas moderados e se aplicando uma escala geográfica mais detalhada, apesar da possibilidade de modesto aumento na precipitação anual, o escoamento superficial deve sofrer, durante a maior parte da estação de cultivo, decréscimo, uma vez que mais precipitação se dará na forma de chuva que de neve. O derretimento da neve ocorrerá mais cedo e acarretará redução na disponibilidade de água quando esta é mais necessária, elevando a demanda para irrigação, pressionando mais os rios e os sistemas de água subterrânea.

Pode-se assumir que a extensão do período de cultivo (ciclo) em consequência das mudanças climáticas terá pequenas alterações nos trópicos e mudanças na precipitação; combinadas com a elevação na demanda para evapotranspiração, aumentam as necessidades de água para irrigação (Fischer et al., 2007).

Döll \& Hauschild (2002), analisaram os impactos potenciais de mudanças climáticas nas necessidades hídricas da irrigação para os Estados do Ceará e Piauí e compararam as necessidades hídricas das culturas de 1996-1998 com o clima de 1951-1980 e de cenários de 2011-2040 (derivados da redução de escala dos modelos climáticos ECHAM4 e HadCM2). Apesar da precipitação média ter projeção de pequena redução caso do ECHAM4, cenários futuros resultarão em significativa redução de demanda, em $48 \%$ para a maioria dos municípios com irrigação (até menos $6,5 \mathrm{~mm}^{-1}$ ) e aumento em apenas $4 \%$ (menos que $0,3 \mathrm{~mm} \mathrm{ano}^{-1}$ ). Os autores atribuíram os resultados à distribuição espacial e estacional da precipitação e questionável redução da evapotranspiração. Com o HadCM2, as reduções das necessidades para irrigação foram ainda maiores. Os autores alertaram não terem modelado a variedade de culturas e consideraram uma taxa fixa de eficiência de uso da água $(0,6)$ concluindo que, possivelmente, o modelo subestimou o uso da água de irrigação devido a uma estimativa conservadora de áreas irrigadas.

Krol et al. (2006) e Krol \& Bronstert (2007) aplicaram o Modelo Regional Integrado para o semiárido nordestino, usando observações diárias de longo prazo, com um cenário constante (sem mudança climática) e tendências climáticas de dois Modelos de Circulação Global, ECHAM4 (Roeckner et al., 1996) e HadCM2 (Johns et al., 1997). Os autores verificaram que projeções de alterações na precipitação sobre a região (2070 2090 comparada a 1961 - 1990) divergiram sendo menos 50\% para ECHAM4 e mais 21\% para HadCM2.

Das diversas fruteiras tropicais irrigadas as bananeiras são plantas muito sensíveis ao estresse hídrico e suas folhas possuem elevado índice de área foliar, o que resulta em alta transpiração; o sistema radicular é superficial, razão pela qual a bananeira é uma espécie que apresenta considerável resposta fisiológica à escassez de água (Vosselen et al., 2005); além do mais, demandam água ao longo de todo o ano por se tratar de cultivo perene com produção constante.

No presente trabalho analisou-se os impactos das mudanças climáticas na necessidade hídrica da cultura da bananeira, considerando-se cenários de mudanças climáticas em escala local (rio Jaguaribe, no trecho compreendido entre as barragens do Castanhão e de Itaiçaba).

\section{Material e MÉTODOS}

A área de domínio foi delimitada entre $4^{\circ} 39^{\prime} 30^{\prime \prime}$ e $5^{\circ} 40^{\prime} 00^{\prime \prime}$ de latitude sul e $37^{\circ} 35^{\prime} 30^{\prime \prime}$ e $38^{\circ} 27^{\prime} 00^{\prime \prime}$ de longitude oeste, ocupando uma área de $6.415,10 \mathrm{~km}^{2}$ (Figura 1) e altitude máxima de aproximadamente $340 \mathrm{~m}$ acima do nível do mar.

Utilizou-se, neste estudo, o sistema integrado de modelagem regional PRECIS (Provendo Clima Regional para Estudos de Impactos), versão 1.2, utilizando-se as condições de contorno do Modelo Climático Regional HadRM3P. O modelo HadRM3P (Modelo Regional Hadley 3) foi implementado no Brasil pelo CPTEC - Centro de Previsão de Tempo e Estudos Climáticos do Instituto Nacional de Pesquisas Espaciais - INPE; sua dinâmica é baseada no HadRM3, uma versão mais recente da componente atmosférica do Modelo de Circulação Global HadCM3 (Johns et al., 2003) do Hadley Centre, Inglaterra.

O modelo regional HadRM3 possui resolução horizontal de $50 \mathrm{~km}$ com 19 níveis na vertical (da superfície até $30 \mathrm{~km}$ na estratosfera) e 4 níveis no solo. A resolução espacial é de 0,44 x $0,44^{\circ}$ latitude/longitude, o que corresponde a uma grade de aproximadamente $50 \mathrm{~km}$. Para fins de mudança climática, o modelo usa uma representação do ciclo de sulfato e de alguns outros aerossóis; maiores detalhes das principais características do HadRM3P e do sistema PRECIS são descritos por Alves (2007).

O Relatório Especial Sobre Cenários de Emissões - SREs (Nakicenovic et al., 2000) definia uma família de cenários, sendo os básicos: 


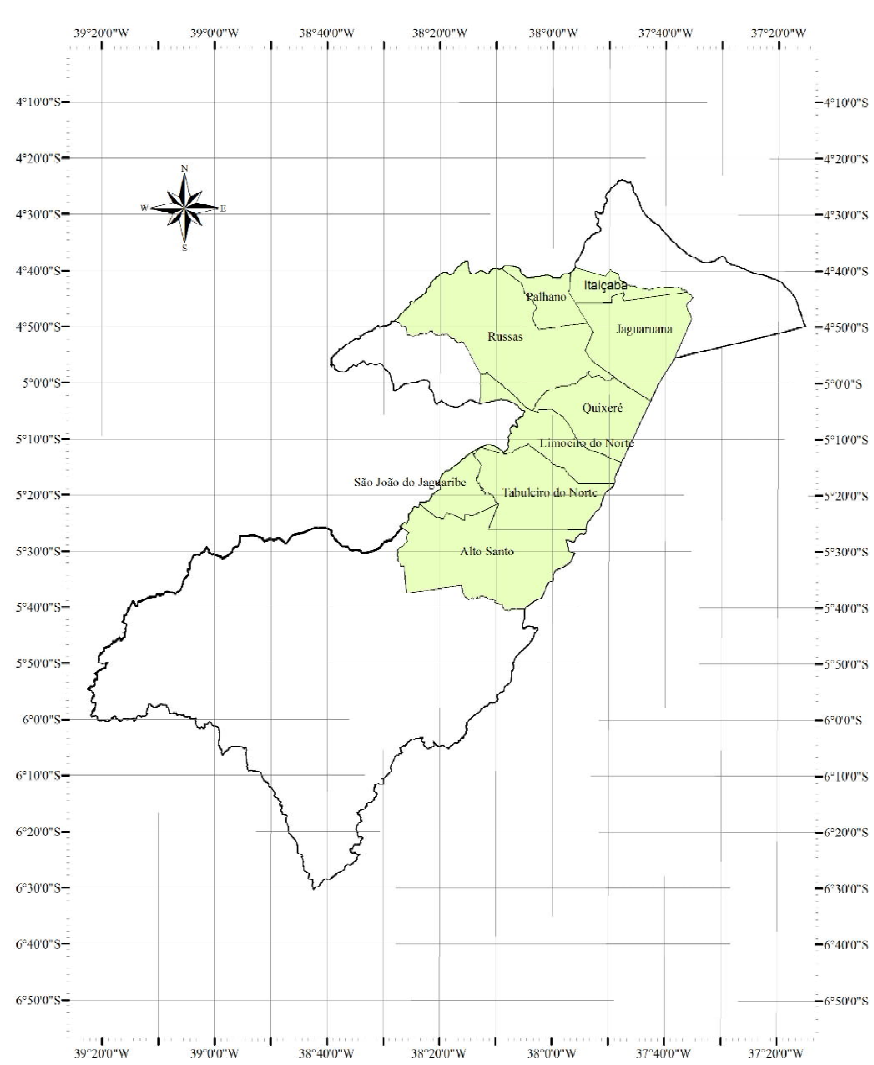

Figura 1. M unicipios e coordenadas geográticas da àrea de estudo

A1: baixo crescimento populacional, crescimento do PIB muito elevado, elevado consumo de energia, baixa mudança no uso da terra, disponibilidade de recursos alta a média, rápido desenvolvimento tecnológico, energia fóssil e balanceada;

A2: alto crescimento populacional, crescimento do PIB médio, elevado consumo de energia, média a alta mudança no uso da terra, disponibilidade de recursos baixa, lento desenvolvimento tecnológico, energia regional;

B1: baixo crescimento populacional, crescimento do PIB elevado, baixo consumo de energia, alta mudança no uso da terra, baixa disponibilidade de recursos, médio desenvolvimento tecnológico, eficiência e introdução de tecnologias limpas;

B2: médio crescimento populacional, crescimento do PIB médio, médio consumo de energia, média mudança no uso da terra, disponibilidade de recursos média, médio desenvolvimento tecnológico, dinâmica energética, como a usual.

Um conjunto de dados composto de uma baseline (climatologia de base) do modelo de 1961-1990 e de projeções climáticas futuras foi gerado no Instituto Nacional de Pesquisas Espaciais - INPE-CPTEC (Alves, 2007). Extraíram-se os dados considerando-se as coordenadas geográficas da região em estudo gerando-se, então, as quadrículas para cada variável, a fim de abranger toda a região estudada.

Tendo em vista que para o setor de irrigação na área do estudo a expansão da área irrigada está sendo visualizada, o horizonte temporal para avaliação dos impactos das mudanças climáticas considerado, foi o ano de 2040.
Uma metodologia similar à aplicada por Knox et al. (1997) para a Inglaterra e País de Gales; Tsanis \& Naoum (2003) para a ilha de Creta, na Grécia; por Victoria et al. (2005) para Santa Catarina, no Brasil foi utilizada para estimar as necessidades de água da bananicultura irrigada em nível de bacia hidrográfica.

Empregou-se um Sistema de Informação Geográfica - SIG, visando à utilização de informação espacial de dados meteorológicos e distribuição dos irrigantes, incorporando-se a variabilidade espacial e temporal das variáveis de demanda de água de irrigação, conforme realizado por Biju et al. (2004) no Uzbekistão e por Fortes et al. (2005) na Índia.

Os dados de saída do modelo foram então interpolados considerando-se a latitude/longitude, através de geoestatística (krigagem) associada a um Sistema de Informação Geográfica. A mesma metodologia foi empregada no Sri Lanka por Silva et al. (2007) e na bacia do Guadalquivir, Espanha, por Díaz et al. (2007) para estimativa de impactos de mudanças climáticas nas necessidades hídricas das culturas irrigadas.

A partir do cadastro de outorga da COGERH (2008), a tipologia da bananicultura irrigada praticada na região (tecnologia empregada e área), foi identificada envolvendo todas as áreas de irrigação difusa (projetos de irrigação fora dos perímetros irrigados) que captam água diretamente no rio, e o Distrito de Irrigação Jaguaribe-Apodi. O trabalho abrangeu um trecho de $160 \mathrm{~km}$ do rio Jaguaribe, envolvendo os seguintes municípios: Alto Santo, Jaguaruana, Limoeiro do Norte, Morada Nova, São João do Jaguaribe, Tabuleiro do Norte, Itaiçaba, Quixeré e Russas, no estado do Ceará.

Aplicou-se um modelo simples de balanço hídrico com precipitação e evapotranspiração mensal enquanto a estimativa da demanda de água para irrigação foi calculada considerandose as diferenças entre evapotranspiração das culturas e precipitação média. A frequência de irrigação requerida para uma cultura em determinado clima, depende sobremaneira da quantidade de água que pode ser armazenada no solo. Assumese que no início das irrigações não haja déficit de umidade no solo (Díaz et al., 2007). A necessidade hídrica líquida das culturas (NHL) pode ser definida como a quantidade de água adicional à umidade no solo proveniente da precipitação que as plantas cultivadas devem receber para se desenvolverem sem estresse hídrico (Fischer et al., 2007), modelada pela Eq. 1:

$$
\mathrm{NHL}_{\mathrm{i}}=\mathrm{ETo}_{\mathrm{i}} \mathrm{Kcfc}-\mathrm{ppt}_{\mathrm{i}}
$$

donde:

$\mathrm{NHL}_{\mathrm{i}}$ - necessidade hídrica líquida ( $\left.\mathrm{mm}\right)$ no mês i

$\mathrm{ETo}_{\mathrm{i}}$ - evapotranspiração de referência $(\mathrm{mm})$ no mês i

Kc - coeficiente de cultivo (adimensional)

fc - fator de cobertura da cultura (adimensional)

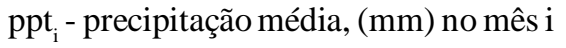

O painel de especialistas da FAO recomendou a adoção do método combinado de Penman-Monteith e ficou conhecido como o método FAO Penman-Monteith, válido globalmente como padrão para cálculo das necessidades hídricas das culturas (Allen et al., 1998). Em situações nas quais se disponha apenas de temperatura do ar, a evapotranspiração de referência 
(ETo) pode ser estimada pela equação de Blaney-Criddle (Doorenbos \& Pruit, 1977).

O fator de cobertura das culturas (fc) considera a superfície foliar que evapotranspira como uma fração da área cultivada, tornando-se função da espécie irrigada e espaçamento adotado, variando com o seu desenvolvimento (Allen et al., 1998); neste trabalho o fc foi considerado igual a 1 para bananeiras, correspondente a $100 \%$ de área sombreada (Freitas et al., 2008).

De vez que a Necessidade Hídrica Líquida (NHL) é igual à evapotranspiração da cultura (ETc), a Necessidade Hídrica Bruta (NHB) foi calculada conforme a Eq. 2, sendo igual à NHL dividida pela eficiência de irrigação para que sejam consideradas as perdas por evaporação, escoamento superficial e percolação profunda. A estimativa de demanda de água para irrigação foi empregada neste estudo em escala de sub-bacia, podendo ser aplicada em diferentes escalas: de bacia (Dantas Neto et al., 2005; Freitas et al., 2008); de perímetro irrigado (Ferreira et al., 2006; Carvalho et al., 2009); ou ainda de parcela irrigada (Figueiredo et al., 2006; Montenegro et al., 2008). A partir das necessidades de irrigação líquidas e da eficiência final de irrigação, foram estimadas, então, as necessidades hídricas brutas (NHB).

$$
\mathrm{NHB}_{\mathrm{i}}=\frac{\mathrm{NHL}_{\mathrm{i}}}{\mathrm{Ea}_{\text {pond. }}}
$$

$\mathrm{NHB}_{\mathrm{i}}$ - necessidade hídrica bruta $(\mathrm{mm})$ no mês i

$\mathrm{Ea}_{\text {pond }}$ - eficiência de aplicação poderada pela área

Também se consideraram os valores de eficiência de aplicação (Ea) alcançados pelos irrigantes da região (Nunes, 2006).

Allen et al. (1998) recomendam, para a bananeira, o uso de valores de Kc de 0,50 e 1,10 para as fases inicial e intermediária durante o primeiro ano de cultivo e 1,00 e 1,20 para as mesmas fases, durante o segundo ano de cultivo. Bassoi et al. (2001), trabalhando com a cultivar Pacovan e utilizando o método do balanço hídrico do solo, na região de Petrolina, PE, encontraram valores médios de 0,70 para a fase vegetativa e 1,10 para a fase de floração/frutificação.

Montenegro et al. (2008), definiram o Kc em Paraipaba, CE, e obtiveram 0,60 do transplantio até 170 dias após o plantio (DAP); em seguida, devido ao crescimento vegetativo das plantas no $1^{\circ}$ ciclo, houve um rápido aumento do Kc até atingir um valor máximo de 1,05 , durante o estádio de florescimento e desenvolvimento dos frutos do $1^{\circ}$ ciclo (240-360 DAP); após a colheita dos cachos e a eliminação das plantas do $1^{\circ}$ ciclo, os valores de Kc decresceram e atingiram um valor médio de 0,86 , no estádio de crescimento vegetativo do $2^{\circ}$ ciclo (415-560 DAP); a seguir, o Kc voltou a subir para 1,05 durante o florescimento e desenvolvimento dos frutos do $2^{\circ}$ ciclo (580-680 DAP).

Considerou-se, neste trabalho, um Kc de 1,1 para cultivos estabilizados após o $2^{\circ}$ ano (Allen et al., 1998), o qual se aproxima dos resultados dos citados autores para a fase de florescimento e desenvolvimento dos frutos.

O modelo climático permitiu que incrementos fossem projetados na temperatura e, consequentemente, elevação na evapotranspiração de referência (ETo) de 3 e de 2,2\% e redução na precipitação de 31 e de $37 \%$ em 2040 para os cenários A2 e $\mathrm{B} 2$, respectivamente.

\section{RESULTADOS E DisCUSSÃO}

O levantamento das áreas irrigadas da bacia permitiu conhecer a tipologia da irrigação da cultura da banana, representada pela área e sistema de irrigação adotado. Verificase que a maior parte da área irrigada para banana adota a irrigação localizada Para utilização de uma eficiência de irrigação para toda a área do estudo, calculou-se um índice único ponderado pela área de cada método de irrigação adotado (Tabela 1).

Tabela 1. D eterminação da eficiência média ponderada pela área de cada méto do de irrigação

\begin{tabular}{lccr}
\hline \multicolumn{1}{c}{$\begin{array}{c}\text { Sistema de } \\
\text { irrigação }\end{array}$} & $\begin{array}{c}\text { Área (ha) } \\
\text { (a) }\end{array}$ & $\begin{array}{c}\text { Eficiência } \\
\text { mensurada (b) }\end{array}$ & \multicolumn{1}{c}{ axb } \\
Inundação & 98,2 & 0,52 & 48,26 \\
Sulco & 61,45 & 0,54 & 33,18 \\
Aspersão & 5,30 & 0,64 & 3,39 \\
Pivot central & 15,75 & 0,65 & 10,23 \\
Localizada & 748,05 & 0,74 & 553,56 \\
\hline Total & 923,35 & & 648,63 \\
\hline Média ponderada & & & 0,70 \\
\hline
\end{tabular}

A Tabela 2 apresenta os valores das projeções das necessidades hídricas brutas (NHB) mensais $(\mathrm{mm})$ para a cultura da banana irrigada, considerando-se a climatologia de base do modelo (1961-1990) e para o ano de 2040, cenários A2 e B2, bem como mínimos, máximos, média e desvio padrão observados espacialmente para a área do estudo. Os valores negativos indicam que não há necessidade de irrigação, sendo a demanda de evapotranpiração totalmente atendida pela precipitação. Projetou-se uma elevação da necessidade hídrica bruta média anual em 2040 com relação às condições iniciais (climatologia de base), de $1.989 \mathrm{~mm}$ para 2.536 e $2.491 \mathrm{~mm}(27,50$ e 25,24\%) para os cenários A2 e B2, respectivamente.

Isto ocorreu devido a um efeito combinado da elevação da evapotranspiração, resultante do aumento da temperatura, agravado pela redução da precipitação, notadamente nos meses chuvosos de março e abril (quando a cultura passaria a ter necessidade de ser irrigada), resultando em aumento das necessidades de irrigação ao longo de todo o ano.

Por outro lado, Medeiros (2003), ao avaliar os impactos das mudanças climáticas utilizando modelo estocástico de precipitação para transferir as alterações na precipitação gerada por um modelo de circulação global da atmosfera na bacia do rio Paraguaçu, Bahia, concluiu que os impactos hidrológicos na bacia do estudo eram mais fortemente controlados pelas reduções na precipitação que pelos acréscimos da temperatura.

A avaliação, entretanto, merece ser continuada utilizando-se outros modelos climáticos a fim de confirmar, especialmente, como a precipitação se comportará na região Nordeste, tal como a capacidade de armazenamento de água em açudes, resultante dos impactos das mudanças climáticas no volume afluente anual. 
Tabela 2. Estimativa da necessidade de irrigação bruta (NHB) mensal $(\mathrm{mm})$ para a cultura da banana irrigada

\begin{tabular}{|c|c|c|c|c|c|c|c|c|c|c|c|c|c|}
\hline & Jan & Fev & Mar & $\mathrm{Abr}$ & Mai & Jun & Jul & Ago & Set & Out & Nov & Dez & Anual \\
\hline $1961-1990$ & & & & & & & & & & & & & \\
\hline $\begin{array}{l}\text { Mínimo } \\
\text { Máximo } \\
\text { Média } \\
\text { Desvio Padrão }\end{array}$ & $\begin{array}{r}-77 \\
138 \\
90 \\
41\end{array}$ & $\begin{array}{r}-35 \\
143 \\
93 \\
36\end{array}$ & $\begin{array}{r}-46 \\
36 \\
4 \\
15\end{array}$ & $\begin{array}{r}-279 \\
55 \\
-12 \\
64\end{array}$ & $\begin{array}{r}-302 \\
165 \\
83 \\
79\end{array}$ & $\begin{array}{r}-170 \\
226 \\
180 \\
56\end{array}$ & $\begin{array}{r}-16 \\
258 \\
234 \\
37\end{array}$ & $\begin{array}{r}120 \\
282 \\
266 \\
19\end{array}$ & $\begin{array}{r}189 \\
277 \\
264 \\
14\end{array}$ & $\begin{array}{r}214 \\
294 \\
280 \\
12\end{array}$ & $\begin{array}{r}199 \\
280 \\
263 \\
13\end{array}$ & $\begin{array}{r}145 \\
270 \\
244 \\
23\end{array}$ & 1.989 \\
\hline $\begin{array}{l}2040 \text { A2 } \\
\text { Mínimo } \\
\text { Máximo } \\
\text { Média } \\
\% \\
\text { Desvio Padrão }\end{array}$ & $\begin{array}{r}73 \\
215 \\
166 \\
84 \\
31\end{array}$ & $\begin{array}{r}43 \\
178 \\
132 \\
42 \\
31\end{array}$ & $\begin{array}{r}-7 \\
131 \\
84 \\
2.000 \\
29\end{array}$ & $\begin{array}{r}-85 \\
157 \\
93 \\
875 \\
50\end{array}$ & $\begin{array}{r}22 \\
220 \\
185 \\
123 \\
37\end{array}$ & $\begin{array}{r}13 \\
257 \\
233 \\
29 \\
31\end{array}$ & $\begin{array}{r}91 \\
276 \\
262 \\
12 \\
20\end{array}$ & $\begin{array}{r}198 \\
287 \\
276 \\
4 \\
11\end{array}$ & $\begin{array}{r}208 \\
286 \\
270 \\
2 \\
15\end{array}$ & $\begin{array}{r}239 \\
299 \\
284 \\
1 \\
11\end{array}$ & $\begin{array}{r}240 \\
290 \\
277 \\
5 \\
11\end{array}$ & $\begin{array}{r}223 \\
289 \\
274 \\
12 \\
13\end{array}$ & $\begin{array}{l}2.536 \\
27,50\end{array}$ \\
\hline $\begin{array}{l}2040 \text { B2 } \\
\text { Mínimo } \\
\text { Máximo } \\
\text { Média } \\
\% \\
\text { Desvio Padrão }\end{array}$ & $\begin{array}{r}79 \\
177 \\
157 \\
74 \\
20\end{array}$ & $\begin{array}{r}58 \\
197 \\
164 \\
76 \\
31\end{array}$ & $\begin{array}{r}-26 \\
84 \\
41 \\
925 \\
26\end{array}$ & $\begin{array}{r}-169 \\
149 \\
92 \\
867 \\
53\end{array}$ & $\begin{array}{r}-49 \\
216 \\
178 \\
114 \\
44\end{array}$ & $\begin{array}{r}48 \\
254 \\
234 \\
30 \\
27\end{array}$ & $\begin{array}{r}75 \\
273 \\
260 \\
11 \\
22\end{array}$ & $\begin{array}{r}131 \\
200 \\
272 \\
2 \\
9\end{array}$ & $\begin{array}{r}141 \\
196 \\
268 \\
2 \\
10\end{array}$ & $\begin{array}{r}246 \\
297 \\
282 \\
1 \\
11\end{array}$ & $\begin{array}{r}239 \\
284 \\
273 \\
4 \\
10\end{array}$ & $\begin{array}{r}212 \\
285 \\
270 \\
11 \\
14\end{array}$ & $\begin{array}{l}2.491 \\
25,24\end{array}$ \\
\hline
\end{tabular}

As Figuras 2A, B e C, agrupam mapas temáticos da necessidade de irrigação bruta total anual estimada para a climatologia de base do modelo e o ano de 2040, cenários A2 e B2, respectivamente. Os mapas foram resultantes da interpolação dos dados georreferenciados, empregando-se a krigagem. Observa-se que a demanda de água para irrigação aumenta no sentido proximidade do Oceano para o interior do semiárido, apresentando-se maior em 2040, no cenário A2.

Freitas et al. (2008) aplicaram a ferramenta de Sistemas de Informação Geográfica, utilizando o inverso do quadrado da distância como interpolador para elaborar mapas temáticos de evapotranspiração da cultura na bacia do São Francisco e encontraram valores de 1.449 a $2.356 \mathrm{~mm}$ anuais, aproximandose dos resultados estimados pelas variáveis da climatologia de base do modelo (média de $1.989 \mathrm{~mm}$ ).

Neste trabalho a metodologia foi empregada para elaboração de mapas temáticos de necessidade de irrigação, considerandose mudanças climáticas. A aplicação de um modelo climático regional associado a um Sistema de Informação Geográfica, permitiu a avaliação de impactos em áreas menores, em comparação com os modelos globais que atuam em nível global e continental, sendo que esta melhoria na resolução espacial possibilitou uma análise mais detalhada dos impactos, em nível local; já a krigagem tornou possível a representação da distribuição espacial da variabilidade e o Sistema de Informação Geográfica, sua visualização em mapas temáticos.

Silva et al. (2007), aplicaram o modelo do Hadley Centre for Climate Prediction (HadCM3) (resolução de 300 km x 300 km) no Sri Lanka, em uma resolução local de $16 \mathrm{~km}$ x $16 \mathrm{~km}$. A demanda de água para irrigação de arroz em 2050 foi prevista aumentar, em média, no País, 23 e 13\% para os cenários A2 e $\mathrm{B} 2$, respectivamente; isto ocorreu devido mais à redução da precipitação durante a época de irrigação (embora a precipitação total anual tenha sido prevista pelo modelo de aumentar), combinada com aumento da evapotranspiração (2 e $1 \%$ para os cenários A2 e B2, respectivamente), decorrente do aumento
A.

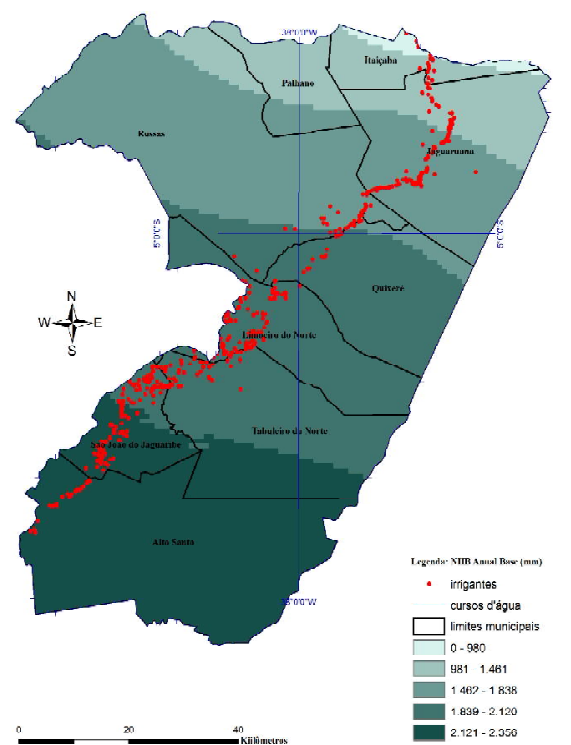

B.

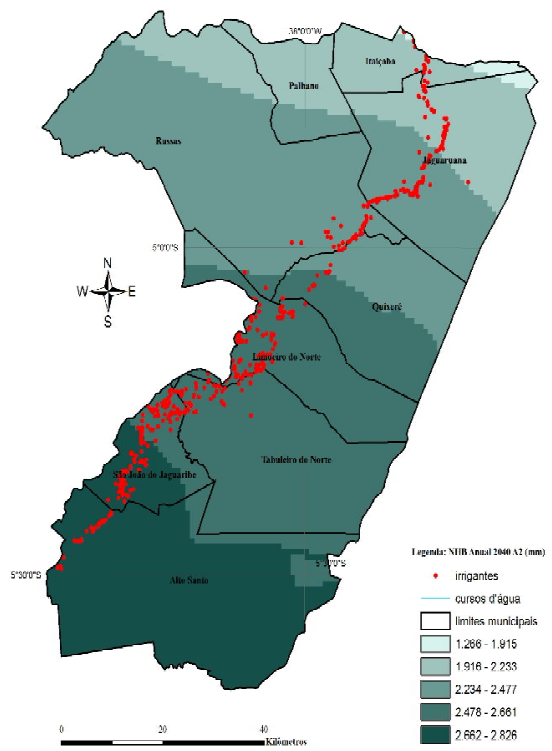

C.

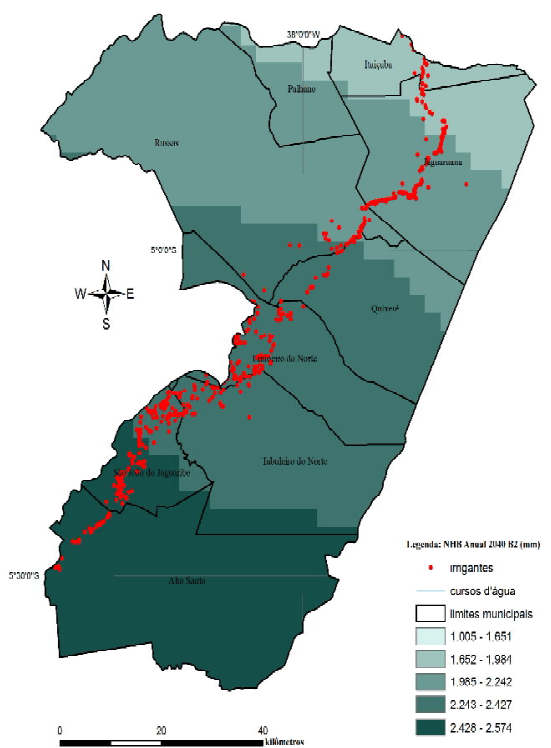

Figura 2. Mapas da distribuição espacial da necessidade de irrigação bruta total anual $(\mathrm{mm}$ ) estimada (A) para a climatologia de base (1961-90); (B) para o ano de 2040, cenário A2; e (C) para o ano de 2040, cenário B2 
da temperatura $\left(1,4\right.$ e $1{ }^{\circ} \mathrm{C}$ para os cenários A2 e B2, respectivamente).

Díaz et al. (2007) estimaram, para 2050, na bacia de Gualdalquivir, Espanha, uma elevação na média das necessidades hídricas de aproximadamente 19,3 e 16,3\%, cenários A2 e B2, respectivamente, causada sobretudo pela redução da precipitação na época de plantio e elastecimento da estação de irrigação.

Mesmo sendo a cultura da banana perene e por se tratar de região semiárida, os resultados apresentados acima se aproximam dos estimados neste trabalho para a região do Jaguaribe $(27,50$ e 25,24\%, para os cenários A2 e B2, respectivamente).

Enquanto a elevação de $\mathrm{CO}_{2}$ tende, sozinha, a acelerar o crescimento das plantas e a produtividade das culturas, temperaturas mais elevadas e mudanças no regime pluviométrico podem não só beneficiar mas também prejudicar os sistemas agrícolas. Aumento na concentração de $\mathrm{CO}_{2}$ pode induzir o fechamento dos estômatos das plantas e, assim, reduzir perdas de água por transpiração (Tubiello \& Ewert, 2002).

Quando a transpiração é reduzida, a perda de calor pelas folhas desacelera e sua temperatura se eleva. Estresse hídrico nas plantas é aumentado por baixa umidade, altas temperaturas, ventos fortes e alta intensidade luminosa; na realidade, incertezas permanecem em virtude da falta de conhecimento em modelos de simulação de plantas (Eitzinger et al., 2003). Por outro lado, uma das restrições do modelo HadRM3P implementado pelo CPTEC utilizado, é a impossibilidade de estimativa futura da evapotranspiração de referência pelo método combinado FAO Penman-Monteith, recomendado pelo painel de experts da FAO, válido globalmente como padrão para cálculo das necessidades hídricas das culturas, uma vez que o mesmo só possibilita a estimativa de temperatura e precipitação; entretanto, torna-se importante a confirmação da tendência de como a temperatura e a precipitação deverão se comportar diante das mudanças climáticas na região e, consequentemente, das necessidades hídricas dos cultivos irrigados, a fim de se planejar medidas adaptativas para os irrigantes, tais como melhorias de eficiência na aplicação da água e escolha de métodos de irrigação e adaptação no padrão de cultivo, selecionando-se culturas que consumam menos água.

Outro aspecto a considerar é a necessidade de melhorias na resolução dos modelos climáticos regionais que permitirão uma avaliação ainda mais precisa dos impactos locais.

\section{Conclusões}

1. As mudanças climáticas, conforme o modelo e cenários processados, impactam na demanda de água para irrigação da cultura da bananeira, como consequência do balanço hídrico determinado pela evapotranspiração e precipitação.

2. Temperaturas mais elevadas causam maior demanda para evapotranspiração das plantas e a tendência flui em direção a um incremento na demanda de água para irrigação, agravada pela redução na precipitação; desta forma, prevê-se que mudança climática funciona como outra fonte de incremento na demanda de água para a agricultura juntamente com a expansão da área irrigada.

\section{LITERATURA CITADA}

Allen, R. G.; Pereira, L. S.; Raes, D.; Smith, M. Crop evapotranspiration: Guidelines for computing crop water requirements. Rome: FAO, 1998. 300p. FAO. Irrigation and Drainage Paper, 56

Alves, L. M. Simulações da variabilidade do clima presente sobre a América do Sul utilizando um modelo climático regional. São José dos Campos: INPE, 2007. 92p. Dissertação Mestrado

Bassoi, L. H.; Silva, J. A. M.; Silva, E. E. da; Ramos, C. M. C.; Targino, E. de L.; Maia, J. L. T.; Ferreira, M. de N. Consumo de água e coeficiente de cultura em bananeira irrigada por microaspersão. Petrolina: Embrapa Semi-Árido, 2001. 4p. Comunicado Técnico, 108.

Biju, A.; George, N. S.; Raghuwanshi, R.; Singh. Development and testing of a GIS integrated irrigation scheduling model. Agricultural Water Management, v.66, p.221-237, 2004.

Binder, L. C. W. Climate change and watershed planning in Washington State. Journal of the American Water Resources Association, v.42, p.915-926, 2006.

Carvalho, M. A. de; Méllo Júnior, A. V. M.; Schardong, A.; Porto, R. L. L. Sistema de suporte à decisão para alocação de água em projetos de irrigação. Revista Brasileira de Engenharia Agrícola e Ambiental. v.13, p.10-17, 2009.

COGERH - Companhia de Gestão dos Recursos Hídricos do Ceará. Cadastro de Outorga, Fortaleza, 2008.

Dantas Neto, F. S.; Costa, J. N. M. da; Soares, A. A.; Sediyama, G. C.; Costa, L. C. Sistema computacional aplicado ao cálculo da demanda hídrica georeferenciada para a cultura o feijão. Revista Brasileira de Engenharia Agrícola e Ambiental. v.9, p.215-221, 2005.

Díaz, J. A. R.; Weatherhead, E. K.; Knox, J. W.; Camacho, E. Climate change impacts on irrigation water requirements in the Guadalquivir river basin in Spain. Regional Environmental Change, v.7, p.149-159, 2007.

Dibike, Y. B.; Coulibaly, P. Hydrologic impact of climate change in the Saguenay watershed: comparison of downscaling methods and hydrologic models. Journal of Hydrology, v.307, p.145-163, 2005.

Döll, P.; Hauschild, M. Model-based regional assessment of water use - an example for semi-arid Northeastern Brazil. Water International, v.27, p.310-320, 2002.

Doorenbos, J.; Pruitt, W. O. Guidelines for predicting crop water requirements. Rome: FAO, 1977. 179p. FAO Irrigation and Drainage Paper, 24

Eitzinger, J.; Stastná, M.; Zalud, Z.; Dubrovsky, M. A simulation study of the effect of soil water balance and water stress on winter wheat production under different climate change scenarios. Agricultural Water Management, v.61, p.195-217, 2003. 
Ferreira, P. A.; Borges Júnior, J. C. F.; Hedden-Dunkhorst, B.; Andrade, C. de L. T. da S. Modelo computacional para suporte à decisão em áreas irrigadas. Parte II: Testes e aplicação. Revista Brasileira de Engenharia Agrícola e Ambiental. v.10, p.783-791, 2006.

Figueiredo, F. P. de; Mantovani, E. C.; Soares, A. A.; Costa, L. C.; Ramos, M. M.; Oliveira, F. G. Produtividade e qualidade da banana prata anã, influenciada por lâminas de água, cultivada no Norte de Minas Gerais. Revista Brasileira de Engenharia Agrícola e Ambiental. v.10, p.798-803, 2006.

Fischer, G.; Tubiello, F. N.; Velthuizen. H. V.; Wiberg, D. A. Climate change impacts on irrigation water requirements: effects of mitigation, 1990-2008. Technological Forecasting \& Social Change, v.74, p.1083-1107, 2007.

Fortes, P. S.; Platonov, A. E.; Pereira, L. S. GISAREG - A GIS based irrigation scheduling simulation model to support improved water use. Agricultural Water Management, v.77, p.159-179, 2005.

Freitas, W. da S.; Ramos, M. M.; Costa, S. L. da. Demanda de irrigação da cultura da banana na bacia do Rio São Francisco. Revista Brasileira de Engenharia Agrícola e Ambiental. v.12, p.343-349, 2008.

Hegerl, G. C.; Zwiers, F. W.; Braconnot, P.; Gillett, N. P.; Luo, Y.; Marengo, O. J. A.; Nicholls, N.; Penner, J. E.; Stott, P. A. 2007: Understanding and attributing climate change. In: Climate change 2007: The physical science basis. Contribution of working group I to the fourth assessment report of the intergovernmental panel on climate change In: Solomon, S.; Qin, D.; Manning, M.; Chen, Z.; Marquis, M.; Averyt, K. B.; Tignor M.; Miller, H. L. (ed.) Intergovernmental Panel on Climate Change, 2007. <http://www.ipcc.ch/pdf/ assessment-report/ar4/wg1/ar4-wg1-chapter9.pdf> 31 Out. 2009.

Johns, T. C.; Carnell, R. E.; Crossley, F. F. Mitchell, J. F. B.; Senior, C. A.; Tett, S. F. B.; Wood, R. A. The second Hadley Centre coupled ocean-atmosphere GCM: Model description, spinup and validation. Climate Dynamics. v.13, p.103-134, 1997.

Johns, T. C.; Gregory, J. M.; Igram, W. J.; Johnson, C. E.; Jones, A. Lowe, A.; Mitchell, J. F. B; Roberts, D. L.; Sexton, D. M. H.; Steveson, D. S.; Tett, S. F. B.; Woodage, M. J. Anthropogenic climate change for 1860 to 2100 simulated with the HadCM3 model under updated emissions scenarios. Climate Dynamics, v.20, p.583-612, 2003.

Knox, J. W.; Weatherhead, E. K.; Bradley, R. I. Mapping the total volumetric irrigation water requirements in England and Wales. Agricultural Water Management, v.33, p.1-18, 1997.

Krol, M. S.; Bronstert, A. Regional integrated modeling of climate change impacts on natural resources and resources usage in semi-arid Northeast Brazil. Environmental Modelling \& Software, v.22, p.259-268, 2007.

Krol, M. S.; Jaeger, A.; Bronstert, A.; Güntner, A. Integrated modeling of climate change, water, soil, agricultural and socialeconomic processes: A general introduction of the methodology and some exemplary results from the semi-arid Northeast of Brazil. Journal of Hydrology, v.328, p.417-431, 2006.
Medeiros, Y. D. P. Análise dos impactos das mudanças climáticas em região semi-árida. Revista Brasileira de Recursos Hídricos, v.8, p.127-136, 2003.

Montenegro, A. A. T.; Gomes, A. R. M.; Miranda, F. R. de; Crisóstomo, L. A. Evapotranspiração e coeficiente de cultivo da banana para a região litorânea do Ceará. Revista Ciência Agronômica. v.39, p.203-208, 2008.

Nakicenovic, N.; Alcamo, J.; Davis, G.; Vries, B. de; Fenhann, J.; Gaffin, S.; Gregory, K.; Grübler, A.; Jung, T. Y.; Kram, T.; La Rovere, E. L.; Michaelis, L.; Mori, S.; Morita, T.; Pepper, W.; Pitcher, H.; Price, L.; Riahi, K.; Roehrl, A.; Rogner, H. H.; Sankovski, A.; Schlesinger, M.; Shukla, P.; Smith, S.; Swart, R.; van Rooijen, S.; Victor, N.; Dadi. Z. Special Report on Emission Scenarios. In: Nakicenovic, N.; Swart, R. (ed.) Intergovernmental Panel on Climate Change, 2000. < http:// www.ipcc.ch/ipccreports/sres/emission/index.htm > 31 Jan. 2007.

Nunes, A. B. de A. Avaliação ex-post da sustentabilidade hídrica e da conservação ambiental de perímetros irrigados implantados - O caso do Perímetro Irrigado Jaguaribe-Apodi (DIJA). Fortaleza: UFC, 2006. 176p. Tese Doutorado

Roeckner, E.; Arpe, K.; Bengtsson, L.; Christoph, M.; Claussen, M.; Dümenil, L.; Esch, M.; Giorgetta, M.; Schlese, U.; Schulzweida, U. The atmospheric general circulation model ECHAM4: Model description and simulation of presentday climate. Hamburgo: Max Planck Institut für Meteorologie, 1996, 90p. Report n.218.

Schlenker, W.; Hanemann, W. M.; Fisher, A. C. Water availability, degree days and the potential impact of climate change on irrigated agriculture in California. Climatic Change, v.81, p.1938, 2007.

Silva, C. S. de; Weatherhead, E. K.; Knox, J. W.; Díaz, J. A. R. Predicting the impacts of climate change - A case study of paddy irrigation water requirements in Sri Lanka. Agricultural Water Management, v.93, p.19-29, 2007.

Tsanis, I. K.; Naoum, S. The effect of spatially distributed meteorological parameters on irrigation water demand assessment. Advances in Water Resources, v.26, p.311-324, 2003.

Tubiello, F. N.; Ewert, F. Stimulating the effects of elevated CO2 on crops: approaches and applications for climate change. European Journal of Agronomy, v.18, p.57-74, 2002.

Victoria, F. B.; Viegas Filho, J. S.; Pereira, L. S.; Teixeira, J. L.; Lana, A. E. Multi-scale modeling for water resources planning and management in rural basins. Agricultural Water Management, v.77, p.4-20, 2005.

Vosselen, V. A.; Verplancke, H.; Ranst, V. E. Assessing water consumption of banana: Traditional versus modelling approach. Agricultural Water Management, v.74, p.201-218, 2005.

Wilby, R. L.; Dawson, C. W.; Barrow, E. M. SDSM - A decision support tool for the assessment of regional climate change impacts. Environmental Modelling \& Software, v.17, p.147$159,2002$. 